\title{
Estrategias
}

\section{de Posicionamiento}

\section{de la Enfermería ante}

los Nuevos Escenarios

de la Salud

Profundas transformaciones han marcado la transición al nuevo milenio. El sorprendente desarrollo científico y tecnológico ha acelerado el proceso de globalización del mundo, ocasionando un mayor intercambio ideológico, cultural y comercial, entre los países.

En este proceso, el escenario mundial de la salud también está experimentando grandes transformaciones. Las reformas globales buscan mejorar la capacidad del Sistema para ofrecer servicios de mejor calidad y lograr una distribución más equitativa de los recursos disponibles. Por otro lado, las organizaciones multilaterales orientadas al desarrollo, actualmente tienen claro que, la salud es un elemento fundamental del desarrollo económico, han dejado atrás la idea de que la economía tiene un impacto sobre la salud, para entender que, se trata de una relación con efecto recíproco (Frenk, 2003).

La Encuesta de Líderes del Sector Salud de Latinoamérica (J\&J, 2007) arrojó que los directores generales de clínicas y hospitales de la región, identifican: 1) que el pensamiento estratégico es el aspecto estructural más importante para el automejoramiento, 2) que las principales oportunidades que ofrece el entorno cambiante son: la posibilidad de expansión de los servicios y la Reforma del Sistema de Salud, 3) que el poder creciente de los terceros pagadores (seguros de gastos médicos, etc.) y las regulaciones gubernamentales son las mayores amenazas. Así mismo, coinciden en que los dos grandes desafíos que enfrentan son la calidad y el costo de los servicios. El estudio plantea entonces la cuestión: ¿Cómo deben actuar los participantes en la cadena de valor de los servicios de salud?

En la Unión Americana se emprendieron varias acciones importantes al respecto:

- La profesionalización de los cuadros directivos de los servicios de salud.

- $\quad$ El mejoramiento continuo de la calidad de los servicios.

- $\quad$ El uso frecuente de consultorías especializadas en costos y en calidad.

- La creación de protocolos clínicos, para controlar la calidad y el costo. 

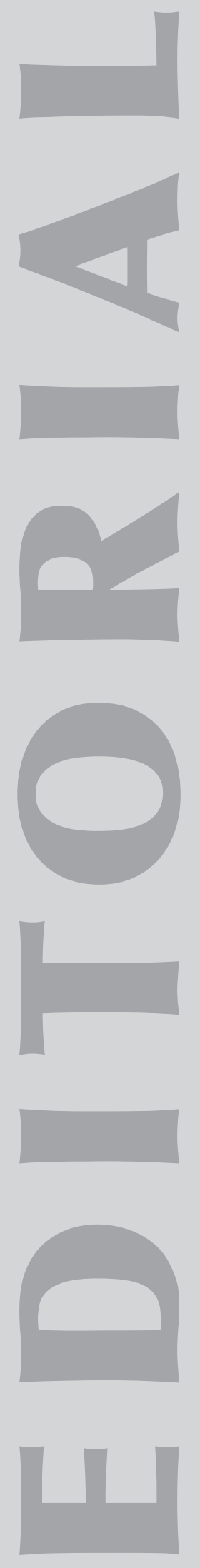

- El fortalecimiento de las Asociaciones y otras organizaciones colegiadas de los médicos.

México se ha caracterizado por grandes cambios económicos, políticos, sociales y culturales, en las últimas décadas. Algunos de ellos son el acelerado proceso de envejecimiento de la población, que ha condicionado una transición epidemiológica. El mayor acceso a la educación básica y a la información, está cambiando el comportamiento de los usuarios, quienes aumentan de manera progresiva y sostenida su participación en el Sistema, porque entienden mejor la salud, la enfermedad y su responsabilidad en la terapéutica. Adicionalmente, conocen sus derechos y están dispuestos a reclamarlos.

Durante los más de 20 años de reforma del sistema de salud en México, los cambios más significativos se han enfocado al proceso de descentralización. Ahora se está iniciando la etapa de la reforma financiera y la construcción de nuevos modelos para financiar los servicios de salud.

En este nuevo escenario, el Sector Privado juega un papel cada vez más significativo en el financiamiento y en la prestación de servicios de salud. Durante años se le restó importancia, sin embargo, desde la década de los setenta, la Medicina Privada ha mostrado un fortalecimiento gradual y sostenido con importantes aportaciones en cuanto a capacidad instalada, recursos humanos, tecnología avanzada y modernización administrativa, entre otros. Contribuyendo así, en favor de la atención a la salud y de la economía del País. La Encuesta Nacional de Salud del 2000 (ENSA, FUNSALUD) mostró que la Medicina Privada atiende al 27\% de la población mexicana. Para el año 2005 la Medicina Privada representó el $30 \%$ de las camas censables, el 25\% de los internamientos, el 35\% de las atenciones ambulatorias del País y el $15 \%$ de los recursos humanos de Enfermería (OCDE, 2005).

Otro aspecto dinámico en el Sistema de Salud es la transformación organizacional. El estudio Prospectiva de la Salud en México, 2005-2050, realizado por la Secretaría de Salud (2004) señala que el hospital, desde la Edad Media, ha sido eje y pieza fundamental del Sistema y que lo seguirá siendo hasta mediados del S. XXI en que nacerá un nuevo paradigma. Habrá un cambio radical en el enfoque actual de atención a la enfermedad, por el de atención a la salud, con el resultante fortalecimiento de la Medicina General y el debilitamiento de las especialidades; la tecnología y los procedimientos asistenciales serán menos invasivos, crecerán los servicios ambulatorios y la atención a distancia, cambiará el concepto de hospital hotel a "ciudades de la salud", las infecciones nosocomiales serán un problema grave y creciente (priones), habrá hospitales albergues para enfermos con infecciones incurables, la tecnología, los insumos y la Administración serán subrogados, la competencia (comercial) se dará entre hospitales públicos y privados, y el profesional de Enfermería tendrá mayor responsabilidad en el plan de tratamiento (Ruelas 2005).

El hospital es reconocido como un Sistema, sus elementos constitutivos son complementarios, interdependientes y sinergizantes, necesitan man- 
tener una eficiente comunicación y la búsqueda de un objetivo común, determinado por las necesidades de su entorno. Pero más allá de esto, el hospital es un Sistema Complejo, sus partes se vinculan mediante una gran cantidad de interconexiones intangibles, multidireccionales y no lineales. En él cohabitan una gran cantidad de culturas diferentes; como la de Enfermería, la de los médicos, los administradores y financieros, entre otros. Todos ellos con lenguajes y valores propios. Es un Sistema de alta complejidad, en virtud de que se desempeña bajo el concepto de economía de redes.

Estos y otros cambios caracterizan el nuevo escenario de los servicios de salud. Sin embargo, aún cuando es referida como uno de los elementos sustanciales del Sistema, Enfermería ha llevado un ritmo de cambio lento. Es indudable que las permanentes iniciativas para la profesionalización han rendido sus frutos; de ser un oficio absolutamente supeditado al trabajo médico, la Enfermería ha logrado espacios de reconocimiento y prestigio. El rumbo debe continuar, pero el ritmo y el modo deben mejorar hasta lograr una reconfiguración total que beneficie a la sociedad, a las instituciones y al mismo gremio.

Ante este nuevo y dinámico escenario, la Enfermería Mexicana enfrenta grandes retos que le exigen nuevas y mayores competencias para tener competitividad en el ámbito profesional. La construcción disciplinar de Enfermería requiere de un profundo compromiso individual y grupal, así como de líderes con poder social e institucional que sepan gestionarlo hasta traducirlo en justicia y desarrollo.

El cambio de la Enfermería no es un fenómeno que pueda darse de manera pasiva, tampoco basta con las mejores intenciones o deseos, ni siquiera es suficiente con ideas brillantes, se requiere visión y acción, como elementos complementarios. La visión es la imagen objetivo del futuro que se desea, implica un conjunto de metas por alcanzar. En la visión siempre subyace un sueño, que por si sólo sirve poco, se necesita el poder que mueva el sueño desde la visión a la acción: una visión sin acción es una alucinación y una acción sin visión es un desastre. Roswyn (2006) dijo: sin poder no hay acción. Esto sólo ocurre compartiendo el sueño con otros y transformándolo de acuerdo a la realidad.

El poder es un concepto ampliamente utilizado con diferentes acepciones, tanto en las ciencias exactas, como en las ciencias sociales. En el campo de la Física por ejemplo, puede referirse a la cantidad de calor que se desprende de un proceso de combustión o a la potencia de una máquina. Michel Foucault lo define como una relación interpersonal de dominio. Sociólogos, psicólogos y administradores lo relacionan con la capacidad de una persona para influir en otras, o para movilizar recursos en la búsqueda de objetivos o metas.

Para Benner el poder incluye la utilización de las prácticas de Cuidado para establecer una relación facultativa (prescriptiva) con los pacientes. Asocia la calidad del poder de Enfermería con el poder transformador y curativo del cuidado proporcionado. 

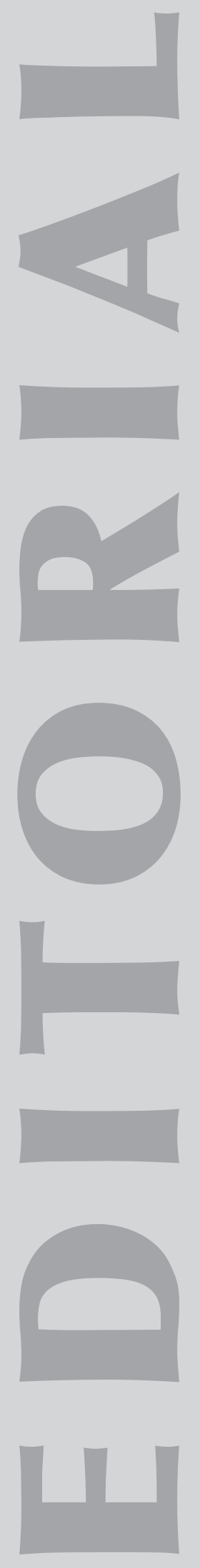

Definitivamente, el poder es un fenómeno complejo, pero es un elemento sine quanon para mejorar la práctica profesional y fortalecer al gremio. Por lo tanto, es necesario identificar cuáles son las fuerzas vivas del entorno y establecer las alianzas necesarias para lograr una Enfermería estratégicamente interconectada y alineada con el contexto en el que se desarrolla.

Hay por lo menos tres aspectos estratégicos en los que Enfermería debe ejercer dominio: 1) control del contenido de la práctica: autonomía para definir el campo disciplinar, dicho llanamente: libertad para hacer lo que uno sabe o para actuar con base en su conocimiento o especialidad, 2) control del contexto de la práctica: participar más activamente, con voz y voto en la administración global de los servicios de salud y 3) control de la capacidad: que se refiere a las competencias profesionales, el conocimiento es precursor, fuente y sostén de poder y de autonomía.

El dominio de la práctica, en sentido amplio, tiene un efecto terapéutico y transformativo en la vida de los pacientes. Pero el dominio no se limita a la experiencia. Dominio sugiere una relación compleja entre formación académica, desarrollo de pensamiento crítico y experiencia (caos y orden).

Partiendo de una perspectiva personal, se identifican al menos tres dimensiones en las que Enfermería puede desarrollar alianzas para crear sus propios espacios de poder:

\section{Alianzas con los pacientes}

2. Alianzas profesionales (internas o gremiales), mediante la creación y/o fortalecimiento de las Redes de Enfermería.

3. Alianzas con los médicos y otros profesionales.

Es necesario cambiar esa anacrónica idea de que la Enfermería es una profesión diferente. Si bien, es importante clarificar y defender su unicidad, también conviene hacer historia comparada con médicos y otros grupos profesionales que han logrado un excelente posicionamiento social. No dividir los esfuerzos, sino sumarlos. Ser capaces de convencer con argumentos válidos, a los médicos y a otros profesionales, incluidos los que ostentan la función de administrar el Sistema y los servicios, que una Enfermería desarrollada es una Enfermería rentable, que las Enfermeras impotentes son Enfermeras ineficaces. Que juntos y por el bien de todos, debemos impulsar el desarrollo de una Profesión que por la función que realiza es estratégica para el éxito del Sistema y el bien común.

Particularmente, los pacientes pueden ser los mejores aliados para lograr el poder que necesita la Enfermería, porque son los clientes del Sistema de Salud, son la sociedad. Ellos representan la perspectiva clínica del Sistema y el poder clínico es la base más sólida de la práctica de la Enfermería. Los pacientes son la pieza esencial que puede hacer la contribución más importante a la base clínica en que se sustenta el poder de la Enfermería. Se justifica plenamente una firme alianza entre pacientes y Enfermeras porque su relación es de interdependencia. 
French y Raven (2007) identifican 6 perspectivas desde las que Enfermería puede crear poder clínico:

1. Poder clínico informativo: el profesional de Enfermería es quien más comparte información con los pacientes (y viceversa) en el equipo de salud. Su relación no sólo es continua la 24 hrs. del día y los 365 días del año. Mantienen un acercamiento integrado, holístico y significativo, que se extiende a la familia y a la comunidad y que dota a la Enfermera de información que cuando es compartida con el resto del equipo, se convierte en una poderosa herramienta en el proceso asistencial.

2. Poder clínico legítimo: que está basado en la formación académica universitaria cada vez más completa, en los niveles básico y de postgrado (formación actualmente reconocida jurídicamente en México), la que emanada de un marco ético aceptado en el que se incluye la defensa de los derechos de los pacientes.

3. Poder clínico carismático: este concepto se relaciona con la motivación interna para inspirar a otros. El poder carismático de la Enfermería tiene que ver con sentir pasión, gusto y orgullo por la Profesión y poder transmitir esa energía positiva, tanto a otros colegas, como a otros integrantes del equipo de salud y a los mismos pacientes al infundirles confianza y la certidumbre de que sus intervenciones profesionales tienen el poder de trasformar sus vidas.

4. Poder clínico extendido o de referencia: se construye a partir de la premisa de que la calidad del cuidado no se logra con un esfuerzo aislado, sino con la participación del equipo, del paciente, de la familia y de la comunidad. El poder extendido se refiere también a la colaboración armónica entre los diferentes turnos y del trabajo en equipos multidisciplinarios. Hace énfasis en la buena comunicación y reconoce la importancia de todos los participantes en la atención del paciente.

5. Poder político: significa la capacidad para diseñar e influenciar en las políticas, así como la oportunidad de determinar la calidad de los procesos y de la vida a la cual tienen derecho las personas.

6. Poder de organización: se refiere a que las Enfermeras puedan diseñar ambientes de trabajo que satisfagan las necesidades de los pacientes y del personal.

Desde la opinión de las autoras, estas versiones sostienen la base del poder clínico de Enfermería. Sin embargo, se tiene poca conciencia de la fuerza que puede representar la alianza entre pacientes y Enfermeras.

La situación actual presenta viejos y nuevos desafíos. La complejidad de la Enfermería contemporánea exige la creación de nuevos patrones de pensamiento, que algunos autores coloquialmente llaman pensamiento "fuera de la caja" (Donley, 2006). Es un pensamiento intelectual, divergente, crítico, opuesto al pensamiento lineal, fuera de los límites convencionales, que se apoya en argumentos teóricos, abstracciones y 

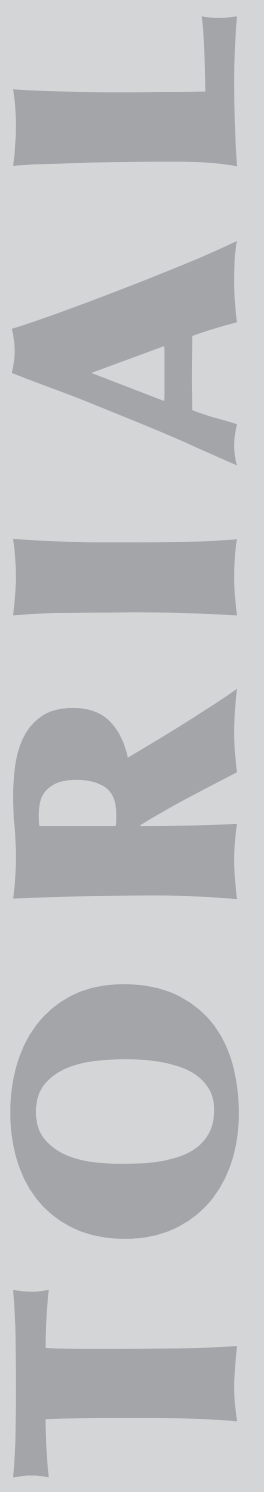

Lic. Ma. Guadalupe Ibarra Castañeda Directora Corporativa del Grupo Ángeles.
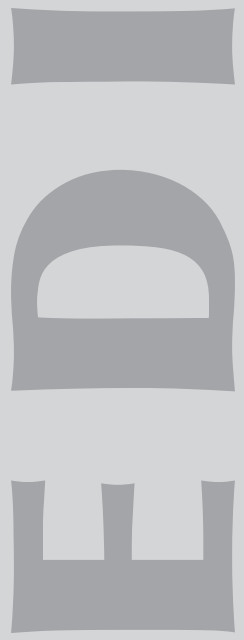

evidencias para tomar decisiones bajo condiciones de ambigüedad. Este tipo de pensamiento valora la capacidad de síntesis por encima de la mente analítica y es la base para promover cambios significativos para construir una Enfermería de Práctica Avanzada.

Crear el nuevo paradigma de la Enfermería necesita de líderes con pensamiento fuera de la caja, con una óptica multidimensional, con la intelectualidad suficiente para desarrollar una visión de futuro, con capacidad para compartirla y con un firme compromiso para emprender las acciones que la operativicen. Pero sobre todo, líderes con nobleza, dispuestos a dar su mayor esfuerzo sin esperar nada personal a cambio, concientes de que su lucha pretende construir la Enfermería de las siguientes décadas, la Enfermería que quizá no verán, la que les proporcionará un mejor futuro a sus colegas de las siguientes generaciones.

Solamente líderes visionarios capaces de ver cómo las cosas pueden ser diferentes, pensadores estratégicos e inspiradores, pueden ser constructores de cultura y poseedores de la poderosa herramienta de gestión que es el poder del conocimiento. Ellos son capaces de mover el sueño. Recordemos el viejo adagio, conocimiento es poder; por ello iMejoremos el cuidado de la salud de la población, mejorándonos a nosotros mismos! 